\title{
Traduire
}

Ine autre perspective sur I t raduction

Revue française de la traduction

245 | 2021

Regards sur l'interprétation

\section{Interpréter en contexte de demande d'asile. Enjeux et pistes de réflexion pour améliorer sa pratique}

\section{Diana Huet de Guerville}

\section{(2) OpenEdition}

1 Journals

\section{Édition électronique}

URL : https://journals.openedition.org/traduire/2454

DOI : 10.4000/traduire.2454

ISSN : 2272-9992

Éditeur

Société française des traducteurs

\section{Édition imprimée}

Date de publication : 5 décembre 2021

Pagination : $67-75$

ISSN : 0395-773X

\section{Référence électronique}

Diana Huet de Guerville, «Interpréter en contexte de demande d'asile. Enjeux et pistes de réflexion pour améliorer sa pratique », Traduire [En ligne], 245 | 2021, mis en ligne le 05 décembre 2021, consulté le 23 décembre 2021. URL : http://journals.openedition.org/traduire/2454 ; DOI : https:// doi.org/10.4000/traduire.2454 


\section{Interpréter en contexte de demande d'asile}

Enjeux et pistes de réflexion pour améliorer sa pratique

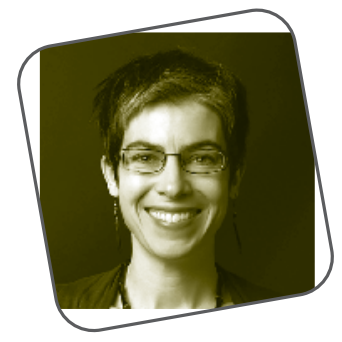

\section{Diana Huet de Guerville}

Traductrice indépendante depuis dix ans, j'ai commencé il y a trois ans l'interprétation par téléphone pour des Nigérianes et Nigérians en demande d'asile, complètement par hasard. N'étant pas formée à l'interprétation, j'ai dû tout apprendre sur le tas, ce qui a généré un certain nombre de difficultés et de questionnements, surtout étant donné la sensibilité du contexte. En cherchant à développer mes compétences en interprétation, je me suis rendu compte qu'il y avait très peu de formations en France en dehors du cadre universitaire. J'ai finalement trouvé une formation en ligne proposée par l'Université de Glasgow, "Interpreting for Refugees: Contexts, Practices and Ethics», que j'ai beaucoup appréciée. J'aimerais ici partager mes expériences d'interprétation en milieu social complexe et proposer des pistes d'amélioration telles que les suggèrent cette formation et mes propres réflexions. 
En 2018, une connaissance, Jeanne ${ }^{1}$, travailleuse sociale, m'a sollicitée pour faire de l'interprétation par téléphone pour une de ses clientes, une demandeuse d'asile nigériane anglophone. J'ai commencé par refuser, en lui expliquant que je n'avais pas de compétences en interprétation. Mais comme elle ne trouvait personne d'autre, elle a insisté, disant que ce serait toujours mieux que rien.

J'ai donc accepté, pour lui rendre service, avant de passer plusieurs jours paniquée à l'idée de faire une prestation pour laquelle je ne me sentais pas du tout qualifiée. J'ai essayé de me préparer au maximum, en me renseignant sur les différentes formes d'interprétation, et en essayant d'en comprendre les techniques. J'en ai assez vite déduit que le téléphone imposait le consécutif, ce qui m'a un peu rassurée. Mais quand j'ai commencé à lire et à regarder des vidéos sur la prise de notes, qui me paraissait trop complexe pour être acquise en quelques jours, je me suis sentie assez dépassée. Je me suis donc concentrée sur une recherche terminologique, ne sachant pas trop ce qui allait véritablement m'être demandé.

Le jour J, j'avais une boule au ventre, mais tout s'est finalement bien passé. Jeanne recueillait le récit de vie de cette demandeuse d'asile pour préparer son dossier auprès de l'Office français de protection des réfugiés et apatrides (OFPRA) - un récit dont le vocabulaire était assez accessible. Le téléphone compliquait certes les choses, car je n'arrivais pas toujours à bien entendre les propos de la personne. J'ai souvent dû lui demander de se rapprocher du combiné, ou de parler plus fort - des injonctions peu évidentes quand votre interlocutrice évoque des sujets très douloureux et intimes (une difficulté plus tard amplifiée par le port du masque). L'accent nigérian est par ailleurs très différent de mon accent étasunien, d'où quelques soucis de compréhension. Mais le plus compliqué était sûrement de devoir couper la personne en plein récit pour ne pas avoir trop à restituer en même temps. Comme elle partageait l'histoire très sensible de son parcours depuis le Nigéria,

1. Prénom modifié pour protéger l'anonymat. 
l'ayant amenée à demander l'asile en France, c'était très compliqué pour moi de lui couper la parole.

J'ai fait du mieux que j'ai pu et, malgré mon épuisement à la fin de cet appel, j'avais l'impression d'avoir réussi à interpréter correctement pour cette personne. Jeanne était contente et reconnaissante, et j'ai surtout eu l'impression d'avoir été réellement et directement utile, ce qui m'arrive rarement avec la traduction de documents. Malgré mon stress avant l'appel, j'ai senti un réel plaisir et une certaine énergie d'avoir ainsi été en contact avec d'autres êtres humains.

J'ai donc continué à accepter des missions pour cette association qui loge des personnes en demande d'asile, car Jeanne a non seulement continué à me solliciter mais elle m'a également recommandée à d'autres personnes dans son service.

Cela fait maintenant trois ans que j'interprète pour cette association, toujours pour des Nigérianes et Nigérians en demande d'asile en France. Ces personnes sont généralement anglophones, sans que l'anglais ne soit nécessairement leur langue maternelle cependant, ce qui peut ajouter à la difficulté de l'interprétation. Le volume d'appels est assez variable (de quelques fois par mois à plusieurs fois dans la même semaine) et le contenu change aussi d'un appel à l'autre: un récit de vie, une préparation pour un entretien avec l'administration française, l'accompagnement pour résoudre une difficulté, la résolution de conflits, une visite de logement, l'explication de règles de vie ou d'un contrat à signer, une réunion avec la maîtresse d'un enfant scolarisé... Je suis à présent davantage habituée à l'accent et aux différences de vocabulaire, et nous pouvons parfois même en rire au téléphone quand il y a un quiproquo. Je connais beaucoup mieux le vocabulaire du système d'asile, même s'il m'arrive encore de devoir faire des recherches sur certains termes. Et, surtout, j'y trouve beaucoup de sens: j'ai l'impression de contribuer à l'aide humanitaire, d'être cohérente avec mes valeurs de solidarité et d'aider des personnes en difficulté de façon concrète.

Mais cela reste un exercice complexe, au-delà de la difficulté inhérente à l'interprétation par téléphone et aux problèmes de compréhension ou de technologie. Après tout, les demandeuses et demandeurs d'asile à l'autre bout du fil ont vécu de grands traumatismes qu'il leur faut répéter à l'envi. Je ne 
trouve pas toujours facile de savoir comment me positionner face à ces situations complexes, ni de trouver la bonne distance. De plus, entendre et transposer ces récits douloureux a naturellement un impact sur moi. Après une interprétation particulièrement lourde (qui évoquait violences, menaces de mort, exploitation, prostitution, excision), Jeanne m'a fait savoir que je pouvais interrompre le récit à tout moment pour indiquer que c'était trop difficile pour moi et demander à la personne de donner moins de détails. J'étais assez surprise, car je n'aurais jamais imaginé limiter la parole de la personne, me voyant comme la «messagère» qui transmet sa parole, peu importe l'impact que cette parole avait sur moi. Mais Jeanne m'a avoué que c'était parfois trop difficile pour elle aussi et que ça lui arrivait de dire qu'il y avait des limites à ce qu'elle pouvait entendre. Et elle me laissait la possibilité d'en faire de même.

Cela m'a fait réfléchir au rôle de l'interprète dans ce genre de situations et m'a fait prendre conscience de mon manque d'expérience et de formation: je ne savais pas réellement ce qui était juste au niveau déontologique. Je peux comprendre le besoin d'une travailleuse sociale de poser des limites, mais cela me semble difficilement justifiable dans mon cas. Après tout, le téléphone crée de la distance, ce qui est frustrant par moments, mais cela a aussi pour conséquence de réduire l'impact des mots. Étant de nature empathique, je crois que je serais beaucoup plus touchée si j'étais en face de la personne. Mais je pense aussi que la pratique de l'interprétation en elle-même bloque un peu l'accès aux émotions: je suis tellement concentrée sur le sens des mots et la façon de les transmettre correctement que je n'ai pas vraiment le temps de me focaliser sur leur contenu. C'est un exercice tellement intellectuel que je ne ressens l'impact émotionnel qu'une fois l'appel terminé.

Car il y a un impact, effectivement. Et j'éprouve souvent le besoin d'en parler aux personnes qui m'entourent pour évacuer. C'est là où le réseau de collègues que j'ai développé grâce à la Société française des traducteurs (SFT) devient primordial. J'ai ainsi pu solliciter des interprètes pour leur demander leur avis et échanger autour de nos expériences. Par exemple, quand j'évoquais ma difficulté à toujours rester à la première personne quand j'interprète, une collègue qui 
travaille avec la police et le tribunal m'a avoué qu'elle n'arrivait plus à dire «je»quand ce que racontait la personne était trop violent pour elle. II y a peut-être effectivement un élément de préservation psychique en jeu. Et cela me rassure de savoir que d'autres interprètes vivent des choses similaires.

Malgré le soutien de mes collègues, j'ai ressenti le besoin de développer mes compétences en interprétation pour être encore plus efficace dans mon travail avec cette association, m'assurer d'adopter les bonnes pratiques, et, à terme, pouvoir chercher d'autres missions d'interprétation dans ce domaine, ou d'autres. Mais j'ai eu beaucoup de mal à trouver des formations continues en France dans le domaine de l'interprétariat pour ce que je recherchais - des techniques de base, surtout en consécutif et de liaison. À mon âge et au stade de ma vie (avec deux enfants à charge), et après m'être déjà endettée pour un premier master, je ne peux pas me permettre de reprendre des études à plein temps, d'autant plus qu'il n'existe pas de programme adéquat là où j'habite.

Je me suis donc penchée sur les formations en ligne. J'ai ainsi découvert une formation gratuite en anglais proposée par l'Université de Glasgow, "Interpreting for Refugees: Contexts, Practices, and Ethics». La formation dure trois semaines (à raison de quatre heures par semaine) et, moyennant 54 euros, permet l'obtention d'un certificat à son rythme (pas de limite dans le temps).

Bien que la formation soit courte, je l'ai trouvée assez complète, avec de nombreux supports (documents, vidéos, mises en scène). La première semaine couvre les enjeux de l'interprétation en contexte humanitaire et le rôle et les compétences de l'interprète; la deuxième aborde les différents environnements professionnels dans lesquels l'interprétation humanitaire peut avoir lieu et donne des conseils pratiques et linguistiques; et la troisième parle de déontologie, de «self-care» (comment ne pas négliger son bien-être en tant qu'interprète humanitaire) et de bonnes pratiques.

Sans détailler tous les apprentissages, qui sont nombreux, cette formation m'a beaucoup appris sur le contexte humanitaire au sens large et a mis en évidence certains éléments à prendre en compte. Par exemple, pour l'interprétation en direct, il est recommandé de s'asseoir en formant un triangle pour que l'interprète affirme sa neutralité et ne prenne pas 
parti. J'appliquerai volontiers ce conseil si je suis amenée à faire ce genre de travail. Étaient aussi énoncées des pratiques de base visant à mettre le ou la bénéficiaire plus à l'aise, comme bien se présenter et expliquer que l'on traduira intégralement tout ce qui est dit.

Avec le recul, je me suis rendu compte que je n'étais pas toujours à l'aise avec la manière dont les séances au téléphone commençaient, avec des présentations très succinctes de la part de la travailleuse sociale - je ne comprenais pas toujours très bien le nom de la personne dont j'allais interpréter les propos et je n'arrivais pas toujours à savoir si j'avais déjà parlé avec elle ou non. Mettant en pratique les recommandations de la formation, j'ai commencé par me présenter, redemander son nom à mon interlocutrice ou interlocuteur et vérifier si on avait déjà travaillé ensemble, puis bien lui expliquer que j'allais tout traduire et m'assurer qu'elle ou il m'entende et me comprenne bien. J'étais aussi gênée par un élément de langage propre au français, I'utilisation de monsieur et madame au lieu du prénom de la personne. Au départ, je traduisais cette adresse en anglais, mais ce n'était pas naturel pour moi, particulièrement dans les cas où il y avait deux personnes (comme un couple en demande d'asile) et où je devais expliciter à qui je m'adressais. J'ai donc ensuite demandé aux personnes comment elles voulaient que je m'adresse à elles, ce qui m'a mise beaucoup plus à l'aise pendant le reste de la conversation (et j'espère qu'il en allait de même pour elles).

La partie de la formation consacrée à la déontologie m'a été fort utile car c'est un sujet très complexe, notamment dans les situations d'interprétation en milieu social. J'ai appris que le code de conduite varie beaucoup d'un pays à l'autre, mais qu'en général très peu de personnes sont au courant de son existence. Bien que j'adhère au code de déontologie de la SFT en tant que traductrice, je n'avais jamais consulté celui relatif à l'interprétation. Et je n'ai que récemment appris qu'il existe une Charte de l'interprétariat médical et social professionnel en France, signée en 2012. Les travailleuses sociales qui me sollicitent ne m'en ont jamais parlé, ce qui me fait penser qu'elles ne la connaissent pas non plus. Nous sommes donc potentiellement deux personnes, dans le triangle asymétrique qu'est l'interprétariat en milieu social, à ne pas 
connaître les bonnes pratiques et le rôle de l'interprète. Cela peut expliquer certains faux pas ou maladresses de ma part, ou de la leur, faute de savoir mieux faire.

Finalement, tout cela a alimenté ma réflexion sur mes devoirs et responsabilités en tant qu'interprète et, plus particulièrement, m'a amenée à reconsidérer l'invitation de Jeanne à interrompre la personne lorsque son récit devenait trop pesant pour moi. J'en conclus qu'il reste de mon devoir de restituer fidèlement tout ce que la personne dit, peu importe la difficulté ou la lourdeur de ce qu'elle décrit. C'est donc à moi de garder une certaine distance émotionnelle, tout en préservant ma propre santé mentale en cherchant du soutien dans mon entourage professionnel ou personnel. Par exemple, une étude de Shakespeare (2012) montre qu'en «devenant la personne qui parle» par l'utilisation de la première personne le temps d'une mission, l'interprète ressent un fort impact émotionnel. Or les interprètes reçoivent en général très peu de soutien, un phénomène encore accentué par leur statut souvent indépendant. Pour l'instant, je n'interviens que quelques heures par semaine - au maximum. Mais si jamais j'augmente mon temps d'interprétation dans ce genre de contextes, il faudra que je veille d'autant plus à trouver des moyens de "self-care» efficaces. La formation suggère diverses méthodes à cet égard (sport, écriture, préparation psychologique, humour, temps avec les proches). La Charte va aussi dans ce sens en incitant les interprètes à développer "au sein de groupes de pairs des capacités d'analyse, de réflexivité et de décentration pour rester à l'écoute et stable dans son travail de traduction».

La formation m'a aussi fait réfléchir à la nécessité de parfois se poser en médiatrice pour fournir des explications culturelles, car la simple traduction pourrait ne pas suffire. La Charte évoque ainsi la responsabilité de l'interprète d'apporter "une attention interculturelle favorisant le repérage d'incompréhensions et de malentendus [...] en proposant - sous forme d'hypothèse à valider - des informations, éclairages ou explications». Je me suis rendu compte que je l'avais fait naturellement au moins une fois, car la travailleuse sociale s'agaçait de ce que la personne avec qui elle discutait d'une erreur assez grave répondait par «no problem» d'un ton qui lui semblait désinvolte. En réponse à 
son énervement grandissant («mais monsieur, c'est en fait un vrai problème!»), je me suis permis d'intervenir en lui expliquant qu'il s'agissait d'une simple tournure exprimant l'acquiescement. En effet, j'avais remarqué que les personnes nigérianes répondaient presque systématiquement "no problem» pour dire «oui, d'accord, je comprends...». Donc il était très clair pour moi que ce monsieur était simplement en train de lui répondre "ovi». Mon explication a permis d'apaiser les choses et d'éviter un conflit stérile. La formation m'a donc permis de comprendre que cet élan de «médiation» culturelle et/ou linguistique a toute son utilité, même si c'est loin d'être un sujet simple.

Ces trois semaines d'apprentissage avec l'Université de Glasgow m'ont été très utiles, mais je constate que j'ai encore énormément à apprendre, surtout si je souhaite aller plus loin dans l'interprétation en milieu social. N'ayant rien trouvé en France, je prévois de m'inscrire à un module "Community Interpreting » en ligne, soit aux États-Unis soit au RoyaumeUni. Ce sont des cursus plus longs, plus poussés et certifiants. J'ai aussi prévu d'aller plus loin grâce à des lectures suggérées au cours de la formation, dont plusieurs documents détaillés proposés par l'ONU (Handbook for Interpreters in Asylum Procedures, Self-Study Module 3: Interpreting in a Refugee Context). Malgré la difficulté de l'interprétation dans ce genre de situation complexe, ce travail me passionne. J'espère donc pouvoir combler cette lacune et voir des formations continues apparaître en France également.

\section{diana.deguerville@yahoo.fr}

Diana HUET DE GUERVILLE est traductrice et interprète franco-américaine née aux États-Unis, de langue maternelle anglaise. Elle traduit du français vers I'anglais depuis 2011, et fait de l'interprétation consécutive et de liaison depuis 2018. Elle est spécialisée dans le domaine public et associatif, l'environnement et le développement international. Installée en France depuis 2006, elle vit désormais dans le Beaujolais, à 20 km au nord de Lyon. 
SHAKESPEARE C. L., Community Interpreters Speaking for Themselves: The Psychological Impact of Working in Mental Health Settings, thèse de doctorat, Hertfordshire (Royaume-Uni), University of Hertfordshire, 2012.

Charte de l'interprétariat médical et social professionnel en France, 2012 : https://www. unaf.fr/IMG/pdf/charte-signee-scan19-12-2012.pdf

UN High Commissioner for Refugees (UNHCR), Handbook for Interpreters in Asylum Procedures, Vienne, UNHCR, 2017: https://www.unhcr.org/dach/ wp-content/uploads/sites/27/2017/09/AUT_Handbook-AsylumInterpreting_en.pdf

Université de Glasgow, Interpreting for Refugees: Contexts, Practices and Ethics: https://www.futurelearn.com/courses/interpreting-for-refugees

UN High Commissioner for Refugees (UNHCR), Self-Study Module 3: Interpreting in a Refugee Context: https://www.refworld.org/docid/49b6314d2.html 